\section{Radiography Studies with Gamma Rays Produced by 14-MeV Fusion Neutrons}

by

CEC 061996

\author{
Donald L. Smith \\ Technology Development Division \\ Argonne National Laboratory \\ Argonne, Illinois 60439 \\ U.S.A. \\ \pm and \\ Yujiro Ikeda and Yoshitomo Uno \\ Fusion Neutronics Laboratory \\ Department of Reactor Engineering \\ Japan Atomic Energy Research Institute \\ Tokai-mura, Ibaraki-ken 319-11 \\ Japan
}

ANH/TD/PP- 84095

\begin{abstract} under contract No. W-31-109ENG-38. Accordingly, the U.S. Government retains a nonexclusive, royalty-free license to publish or reproduce the published form of this contribution, or allow others to do so, for U. S. Government purposes.
\end{abstract}

The submitted manuscript has been authored by contractor of the U.S. Government

Oxygen contained in pure water has been activated via the ${ }^{16} \mathrm{O}(\mathrm{n}, \mathrm{p})^{16} \mathrm{~N}$ reaction using $14-\mathrm{MeV}$ neutrons produced at a neutron generator with the ${ }^{3} \mathrm{H}(\mathrm{d}, \mathrm{n})^{4} \mathrm{He}$ source. Photons of 6.129 and 7.115 $\mathrm{MeV}$, generated by the decay of $7.13-$ second ${ }^{16} \mathrm{~N}$, were then used to demonstrate the feasibility of employing highly penetrating, nearly monoenergetic gamma rays for radiography studies of thick, dense objects composed of elements with medium to relatively high atomic numbers. A simple radiography apparatus was constructed by circulating water continuously between a position near the target of the neutron generator and a remote location where photon transmission measurements were conducted. A collimated photon source and detector arrangement was assembled using lead bricks. 


\section{DISCLAMMER}

Portions of this document may be illegible in electronic image products. Images are produced from the best available original document. 


\section{DISCLAIMER}

This report was prepared as an account of work sponsored by an agency of the United States Government. Neither the United States Government nor any agency thereof, nor any of their employees, make any warranty, express or implied, or assumes any legal liability or responsibility for the accuracy, completeness, or usefulness of any information, apparatus, product, or process disclosed, or represents that its use would not infringe privately owned rights. Reference herein to any specific commercial product, process, or service by trade name, trademark, manufacturer, or otherwise does not necessarily constitute or imply its endorsement, recommendation, or favoring by the United States Government or any agency thereof. The views and opinions of authors expressed herein do not necessarily state or reflect those of the United States Government or any agency thereof. 
A sodium iodide scintillator was employed to detect the photons, and transmission data were recorded with conventional electronics apparatus and a computer. Pulses equivalent to photon energies smaller than $2.506 \mathrm{MeV}$ (corresponding to the cascade sum of $1.333-$ and $1.173-\mathrm{MeV}$ gamma rays from the decay of 5.271 -year ${ }^{60} \mathrm{Co}$ ) were rejected by the electronics settings in order to reduce background and improve the signal-to-noise (S/N) ratio. Respectable $\mathrm{S} / \mathrm{N}$ ratios on the order of 20-to-1 were achieved with this setup, even though very little effort was invested in optimizing the experimental configuration. Most of the background $(\mathrm{N})$ could be attributed to ambient environmental radiation and cosmic-ray interactions with the lead shielding and detector, since it was present even when the accelerator was not operating. Four representative objects were examined by photon radiography in this study. This demonstrated how such interesting features as hidden holes and discontinuities in atomic number could be easily identified from observed variations in the intensity of transmitted photons when these objects were scanned with a collimated beam of gamma rays. Spatial resolutions consistent with the geometry of this simple apparatus were observed in all the examined cases. Some advantages of this technique are described, and potential applications are suggested for a future scenario where fusion reactors are used to generate electric power and very intense sources of high-energy photons from ${ }^{16} \mathrm{~N}$ decay are continuously available as a byproduct of the reactor cooling process. 


\section{Introduction}

The idea of using penetrating photons to examine the interior regions of objects that cannot be observed directly is about 100 years old [1]. The revolutionary discovery of X-rays by Roentgen in 1895 promptly led to the development of non-destructive, non-invasive interrogation techniques applicable to all sorts of objects including the human body. Since the time of Roentgen, this method has developed enormously and now finds routine applications in practically every aspect of modern life, e.g., manufacturing, construction, quality control, medicine, defense, transportation, security, and basic and applied research. The fundamental principles of radiography are described in the literature (e.g., refs. 2-5). The most widely used approach involves $\mathrm{X}$-rays in the range of a few $\mathrm{keV}$ to several hundred $\mathrm{keV}$ that are produced at relatively low cost by electron bombardment of medium to high atomic number metals in sealed, evacuated tubes. While this approach is extremely versatile, there are limits based on the penetrating capacity of these photons. Photons of higher energy and greater penetrating capacity can be obtained from radioactive gamma-ray sources (e.g., ${ }^{60} \mathrm{Co}$ or ${ }^{137} \mathrm{Cs}$ ) and from electron accelerators such as linacs and synchrotrons. Radioactive sources are difficult to handle and store safely. Also, the range of geometrical configurations that are possible with these sources is somewhat limited. Accelerator sources are capable of producing very high radiation intensities and relatively high photon energies, but they always involve continuous-energy photon spectra and the machines are rather costly to build and operate. Because photon transmission through matter is strongly energy dependent, radiography with continuous-energy sources is often beset with problems associated with achieving adequate contrast and selection of proper exposures. Consequently, there is a need for radiography applications to develop a source of photons with the following properties: 
i) monoenergetic (or nearly so), ii) variable intensity, iii) highly penetrating, iv) flexibility in configuring the geometry, v) relatively inexpensive, and vi) relatively safe to handle. The present investigation demonstrates that photons produced by the bombardment of pure water with $14-\mathrm{MeV}$ neutrons satisfy these various criteria quite well. The ${ }^{16} \mathrm{O}(\mathrm{n}, \mathrm{p}){ }^{16} \mathrm{~N}$ reaction produces ${ }^{16} \mathrm{~N}$ which in turn decays fairly quickly, emitting 6.129- and 7.115-MeV gamma rays that can be used for radiography purposes. This paper reviews the physical principles involved in the method, describes a simple experimental apparatus that has been assembled to test the concept, discusses the transmission measurements, and presents the results of radiography investigations of several representative objects. The present work clearly demonstrates how interesting features such as holes and discontinuities in atomic number can be observed through interactions of these high energy, monoenergetic photons with the scanned objects. The advantages of this method are discussed and some potential applications are described for a future scenario where fusion reactors are used to generate electric power, and very intense sources of these photons are therefore continuously available as a byproduct from the reactor cooling process.

\section{Physical Principles}

The physical principles associated with production of photons by neutron-induced reactions which generate radioactive byproducts, and the transmission of these radiations through matter, are well known. The purpose of this section is to review these topics in the context of the ${ }^{16} \mathrm{O}(\mathrm{n}, \mathrm{p}){ }^{16} \mathrm{~N}$ reaction which is of particular interest for the present work. 


\subsection{Photon production}

The ${ }^{16} \mathrm{O}(\mathrm{n}, \mathrm{p}){ }^{16} \mathrm{~N}$ reaction can lead to activation of ordinarily benign pure water $\left(\mathrm{H}_{2} \mathrm{O}\right)$ when it is bombarded with sufficiently energetic neutrons. The natural isotopic abundance of ${ }^{16} \mathrm{O}$ is $99.76 \%$ [6]. The Q-value for this reaction is $-9.637 \mathrm{MeV}$ and that corresponds to a relatively high neutron reaction threshold energy of $10.245 \mathrm{MeV}$ [6]. The reaction cross section is therefore negligible below $11 \mathrm{MeV}$ but increases rapidly to around 80 millibarns near $12 \mathrm{MeV}$, apparently due to a resonance near threshold. The cross section is around 40-50 millibarns in the range 14-15 MeV [7]. The decay by beta $(\beta)$ emission of the product nucleus ${ }^{16} \mathrm{~N}$ with a 7.13 second half life to ${ }^{16} \mathrm{O}$ is a very energetic process [8]. The transition to the ground state of ${ }^{16} \mathrm{O}$ involves beta particles with energies up to $10.419 \mathrm{MeV}$. There are also beta-decay transitions to excited levels of ${ }^{16} \mathrm{O}$ followed by gamma-ray emission. The average energy of the composite beta spectrum is $2.693 \mathrm{MeV}$. Of interest to us in the present context is the fact that $68.8 \%$ of all decays of ${ }^{16} \mathrm{~N}$ produce a $6.129-\mathrm{MeV}$ gamma ray while $4.7 \%$ produce a $7.115-\mathrm{MeV}$ gamma ray. Therefore, water which is activated by sufficiently high energy neutrons becomes a source of nearly monoenergetic high-energy gamma rays which can be employed for a variety of purposes. In this investigation we demonstrate that they can be used for photon radiography.

The limiting conversion efficiency for $14-\mathrm{MeV}$ neutrons to $6.129+7.115 \mathrm{MeV}$ photons in an infinite water medium is approximately the ratio of the ${ }^{16} \mathrm{O}(\mathrm{n}, \mathrm{p}){ }^{16} \mathrm{~N}$ reaction cross section $(40-50$ millibarns) to the neutron total cross section for water (about 3 barns) multiplied by the photonemission branching factor (about 0.74 ). This amounts to an efficiency of about $1 \%$ which is not large 
but nevertheless leads to quite high gamma-ray yields when water is exposed to intense high-energy neutron fields such as those produced by a neutron generator or in a fusion reactor. Recent calculations by Sato et al. for the design of ITER (International Thermonuclear Experimental Reactor) indicate that ${ }^{16} \mathrm{~N}$ activities in the range $0.1-1$ Curie per milliliter of cooling water could be generated during operation of this device [9]. The 14-MeV neutron fields produced by neutron generators are much less intense than those anticipated for plasma fusion devices such as ITER. However, we have been able to demonstrate in the present investigation that sufficient numbers of

${ }^{16} \mathrm{~N}$ gamma rays can be produced with a neutron generator to enable photon radiography to be carried out with moderate resolution by this method. Through engineering design improvements, considerable enhancement of ${ }^{16} \mathrm{~N}$ gamma-ray production could be achieved with neutron generators, relative to the arrangement used in the present investigation. The much higher neutron intensities which may be achieved in the future with plasma sources will afford even better resolution, shorter exposure times and greater flexibility in application of the technique, but will not alter the basic concepts discussed in this paper.

\subsection{Photon transmission}

The transmission of photons through matter is governed by the exponential law [10]. Thus, for a slab of pure elemental material,

$$
I=I_{0} \exp \left[-\left(\text { density in grams } / \mathrm{cm}^{3}\right)(C M S Q / G) \sigma_{\gamma, \text { tor }}\left(E_{\gamma}\right) x\right] \text {, }
$$


where $I_{0}$ is the incident photon intensity, $\mathrm{I}$ is the transmitted photon intensity, CMSQ/G is a factor which depends on the material and is tabulated in Storm and Israel [11], $\sigma_{\gamma, \text { tor }}\left(\mathrm{E}_{\gamma}\right)$ is the photon total cross section in barns for energy $E_{\gamma}$, and $x$ is the thickness of the slab in centimeters. For example, if we apply this formula to the case of $E_{Y}=6 \mathrm{MeV}$, pure iron $(\mathrm{Fe})$ and a $1-\mathrm{cm}$-thick slab we have density $=7.874$ grams $/ \mathrm{cm}^{3}, \mathrm{CMSQ} / \mathrm{G}=0.010780, \sigma_{\gamma, \text { tot }}\left(\mathrm{E}_{\gamma}\right)=2.83 \mathrm{barn}$, and therefore obtain $\mathrm{I} / \mathrm{I}_{0}$ $=0.7865$. The formula can be extended easily to handle multiple slabs, mixtures or compounds. Energy-dependent photon crosss sections can be found in tables, e.g., Storm and Israel [11]. Table 1 shows how penetrating the 6-MeV photons actually are for typical elements. For example, one can expect nearly $10 \%$ of the incident photons to pass through a 10 -centimeter thick slab of iron metal.

\section{Experimental Apparatus}

\subsection{Neutron Source}

The present experiment was carried out at the Fusion Neutron Source (FNS) accelerator located at the Japan Atomic Energy Research Institute (JAERI) in Tokai, Japan [12]. At this facility, deuterons can be accelerated up to $350-\mathrm{keV}$ energy, with beam currents up to 20 milliamperes. The deuterons impinge upon a titanium-tritide target to produce neutrons via the ${ }^{3} \mathrm{H}(\mathrm{d}, \mathrm{n})^{4} \mathrm{He}$ reaction. This arrangement leads to neutron production up to $3 \times 10^{12}$ neutrons per second (into $4 \pi$ steradian). Since the reaction Q-value is $17.591 \mathrm{MeV}$ [6], the energies of the emitted neutrons are in the range 13.3-15 MeV, depending upon the angle of emission relative to the incident deuterons. As neutron generators go, this is a very powerful facility. Consequently, it was possible to carry out the present 
experiment without paying much attention to optimization of the geometrical coupling between the neutron source and water that was being activated for radiography purposes.

\subsection{Radiography apparatus}

The apparatus which was assembled to demonstrate the feasibility of performing radiography with high energy photons from. ${ }^{16} \mathrm{~N}$ decay is shown schematically in Figs. 1 and 2. A circulating loop of water was set up using plastic tubing with about 1 centimeter internal diameter, a water pump and a flow meter, as indicated in Fig. 1. The tubing which contained the flowing water passed in a straight line along a path about 15 centimeters from the point neutron source (accelerator target) at the closest approach. Therefore, for the flow rates we used, any individual volume element of water spent no more than about 0.1 second in the high-fluence region near the target. Since this is a much shorter time than the ${ }^{16} \mathrm{~N}$ half life, the activity generated in the water was always far short of saturation. The physical parameters available for optimization of the irradiation configuration are dwell time and solid angle relative to the point neutron source. We estimate that by coiling the water line and placing it closer to the target it could be possible to achieve ${ }^{16} \mathrm{~N}$ concentrations in the flowing water about two orders of magnitude higher than were attained in the present experiment. We did not explore this possibility because it was not necessary for the purpose of demonstrating the technique. A maximum flow rate of about 10 liters per minute (corresponding to about 2 meters per second velocity in the tubing) could be achieved with the pump available. We found that this particular flow rate fortuitously provided nearly the highest possible intensity of ${ }^{16} \mathrm{~N}$ activity at the position of the radiography apparatus (about 25 meters from the accelerator target location). The ${ }^{16} \mathrm{~N}$ activity in the water 
decreased unavoidably to about $30 \%$ of the value near the accelerator target due to radioactive decay during the required transit time of approximately 12 seconds between the target and the radiography apparatus. However, adequate activity remained at this position to perform the studies reported in this paper.

The specific components of the apparatus which was employed for photon transmission measurements are is shown in Fig. 2. At this position the water line was completely surrounded by lead bricks to a thickness of at least 10 centimeters, except for a single collimator slot 10 centimeters wide by 2.5 centimeters high through which the photons could emerge. A 20-centimeter gap between the shielded source and detector was provided for placement of the objects that were studied by radiography. The photon detector was a 12.7-centimeter-diameter $\times$ 5.2-centimeter-thick sodium iodide scintillator. It was also surrounded by at least 10 centimeters of lead except for a single slot 13 centimeters wide by 2.5 centimeters high which was aligned just opposite to the slot which defined the photon beam. It can be noted from Table 1 that 10 centimeters of lead shielding limits transmission of 6-MeV photons to less than $1 \%$. A rectangular-slot geometry was selected because that provided much greater sensitivity than a cylindrical or square collimator arrangement without sacrificing resolution in the direction along which the objects were actually scanned in this experiment. No attempt was made in the present investigation to carry out two-dimensional scans for any of the examined objects. It would be possible, in principle, to do so if higher concentrations of ${ }^{16} \mathrm{~N}$ were available in the flowing water. As indicated above, significant enhancements of the photon source strength could have been obtained with relatively little difficulty at the FNS laboratory if it had been necessary to do so to obtain adequate sensitivity for our investigation. 
When a rectangular collimator configuration is used, photons will pass through the object at various angles. However, for the present configuration the range of angles due to this effect was relatively small, i.e., $<14$ degrees, and this corresponded to a variation of less than $3 \%$ in path length through the investigated objects.

\subsection{Electronics and data acquisition components

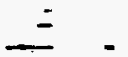

The electronics apparatus for the detector system was very simple. It consisted of a photomultiplier tube, preamplifier, high-voltage power supply, amplifier, delay amplifier, pulse selector and linear gate. The latter three components allowed pulses below an equivalent photon energy of $2.506 \mathrm{MeV}$ to be rejected. Signals corresponding to higher-energy gamma rays were acquired on line with a computer, though it would be possible alternatively to use a multichannel analyzer or even a scaler to record the data. Fig. 3 shows a typical sodium iodide scintillation detector spectrum produced by $6.129+7.115 \mathrm{MeV}$ gamma rays from the radioactive water, as seen by the detector through the above-mentioned collimator system (with no intervening object present).

\section{Radiography Measurements}

\subsection{Experimental procedure}

Four test objects were prepared for use in demonstrating the feasibility of performing 
radiography with this apparatus. Object "A" (shown in Fig. 4) was simply a 5 centimeter $x 15$ centimeter $\times 20$ centimeter rectangular block of stainless steel (mostly iron). Object "B" (shown in Fig. 5) was identical to Object "A" except for a 2-centimeter-diameter hole drilled through the center along its axis. Object "C" (shown in Fig. 6) consisted of two 1-centimeter-thick copper plates with a hidden rectangular lead block 2.5 centimeters $\times 2.5$ centimeters situated between them. Object " $D$ " (shown in Fig. 7) consisted of two 5 centimeter $\times 5$ centimeter $\times 20$ centimeter stainless steel blocks and one pure lead block of the same dimensions stacked together. Each of these distinct objects was scanned in the collimated photon beam, normally in steps of 0.5 centimeter, across a range of about 10 centimeters that fully encompassed the interesting features of the object. Measurements were made periodically with no object in place (100\% transmission). A gamma-ray spectrum was recorded at each position. A record was also made of the accumulated neutron output from the accelerator during each measurement interval since the production rate of ${ }^{16} \mathrm{~N}$ decay photons is directly proportional to the neutron field intensity for a steady-state condition of water flow in the system. The neutron fluence data were used to normalize each measurement. The exposure times for each position were generally about 5 minutes. Therefore, it took about an hour to scan each object and thereby generate the desired radiograph which displayed its characteristic features.

Additional measurements were performed at various times during the experiment to determine the extent and origin of the background. One such set of measurements was made with a 10centimeter-thick lead brick blocking the collimator that defined the photon source. Spectral data were also acquired with the water flow turned off (so no ${ }^{16} \mathrm{~N}$ activity was transported from the target area) and with the FNS accelerator turned off (to determine ambient and cosmic ray background). These 
measurements showed that the signal-to-noise ratio for the experimental setup used was about 20-to1, and that a significant portion of the background came from ambient sources and cosmic ray interactions. It was also found that there was little change in the shape of the spectrum produced by the ${ }^{16} \mathrm{~N}$ gamma rays when various objects were placed between the gamma-ray source and detector for radiography investigation. In other words, although the spectrum yield was reduced the actual appearance of the spectrum was not noticeably distorted by transmission of the gamma rays through the various materials considered. This result served to indicate that most of the detected gamma rays were either primary ones or those that had experienced at most only small angle scattering which did not significantly alter their energies.

\subsection{Analysis of the transmission data}

The events recorded in each spectrum produced from the sodium iodide scintillation detector were summed from just above the lower-level cutoff defined by the pulse selector and linear gate to just below the position where the amplifier saturated. These spectral sums constituted the raw transmission data. This simple approach to the analysis of our experimental data was possible because the shape of the spectrum was not noticeably altered by transmission of photons through the studied objects. These summed counts were corrected for recording dead time, and were further adjusted for exposure time and neutron fluence to yield values of relative transmitted photon intensity I as in Eqn. (1). The relative integrated neutron fluence for each measurement time interval was deduced from the output of a fission chamber monitor located at a fixed position in the target area. Periodic measurements of the gamma ray spectra with no object present defined the equivalent incident photon 
intensity $I_{0}$ so that a meaningful transmission ratio $I / I_{0}$ could be calculated. The desired onedimensional radiographs for the various investigated objects were constructed from these ratios.

\subsection{Results}

The results of ourinvestigation are given in Fig. 8. This figure shows plots of one-dimensional scans of the photon transmission $\mathrm{I} / \mathrm{I}_{0}$ for Objects "A", "B", "C", and "D" (see Figs. 4 - 7). The indicated uncertainties are based on the combined statistics for the summed counts from the sodium iodide scintillation detector spectra and for the neutron flux monitor counts. The data points are connected with solid lines to provide eye guides. The dotted-line segments indicate values of the transmission which were calculated using Eqn. (1) in combination with photon cross sections from Storm and Israel [11] and pertinent material parameters. Qualitative agreement is observed in regions where the transmission is "flat" versus scan distance. However, precise agreement should not be expected because of uncertainties in the density, thickness and composition of the materials involved, and the effects of small-angle photon scattering. Most of the data were acquired in increments of 0.5 centimeter along the direction of scan. Scanning was accomplished by moving the investigated object past the fixed collimator system. It is clear from the data that the spatial resolution observed for these relatively primitive radiographs is consistent with the dimensions of the collimator arrangement. As indicated above, the collimator geometry could have been easily tightened to provide much better resolution if the coupling of the water loop to the neutron radiation field had been optimized. In fact, with a factor of two orders of magnitude enhancement in gamma-ray source strength, which appears to be quite feasible, it would be possible to reduce the collimator dimensions to 0.5 centimeter $\times 0.5$ 
centimeter and achieve the same statistical precision in the data for a given exposure time. Twodimensional scans would be feasible using such a rectangular collimator, but an array of several smaller detectors would be necessary to permit radiographs to be generated in a more reasonable time than would be needed for a single large-detector arrangement like the one used in the present experiment. It is clear that these are engineering design issues which involve no changes in the fundamental principles of this method.

It is obvious from the plots in Fig. 8 that all the interesting features of the investigated objects (see Figs. $4-7$ ) were clearly identified by the one-dimensional photon scans performed in this experiment. Object " $\mathrm{A}$ " is uniform with no distinguishing features. This is evident from its featureless one-dimensional radiograph. Object "B" has a hidden hole which is seen clearly. Likewise, the lead block hidden behind two copper plates in Object "C" leaves an unmistakable imprint on its associated radiograph. Finally, the iron-lead-iron discontinuity which characterizes Object " $\mathrm{D}$ " is also well illustrated by its accompanying radiograph.

\section{Advantages of the Technique}

Six desirable features for a photon radiography system are listed in Section 1 . The present section examines the extent to which these are satisfied by our present approach. The $6.129-\mathrm{MeV}$ gamma rays outnumber those of $7.115 \mathrm{MeV}$ by nearly 15 -to- 1 . Furthermore, the transmission cross sections for these two energies differ by only a few percent across the Periodic Table. Therefore, for all practical purposes, ${ }^{16} \mathrm{~N}$ is a "monoenergetic" photon source and Item (i) is satisfied. Item (ii) is 
clearly satisfied because one can adjust the intensity of the photon field by altering the coupling of the circulating water loop to the neutron radiation field or, more simply, by just changing the flow rate of water in this loop. One can literally "fine tune" the photon intensity by turning a valve (the water flow control). Photons in the 6-7 MeV range are clearly very penetrating so Item (iii) is satisfied. Item (iv) is satisfied because the ${ }^{16} \mathrm{~N}$ activity is transported by water and it is very easy to handle the water and tailor the geometry of a radiography facility based on this technique. This is especially apparent when one considers how difficult it is to deal with sealed, long-lived gamma-ray sources such as ${ }^{60} \mathrm{Co}$ and ${ }^{137} \mathrm{Cs}$. The greatest expense associated with this method involves providing an intense neutron source. Nevertheless, 14-MeV neutron generators are relatively inexpensive on a "per-neutron" basis. Although it is not likely that expensive plasma fusion devices would be built solely for radiography applications, it would be possible to obtain intense photon fields for ${ }^{16} \mathrm{~N}$ for radiography as a byproduct of the process of cooling a D-T fusion machine which is used primarily for energy production. Therefore, we suggest that Item (v) is reasonably well satisfied. Finally, this approach is inherently safe because the ${ }^{16} \mathrm{~N}$ activity generated in and transported by water decays to a negligible level a few minutes after neutron irradiation leaving only essentially benign water which presents virtually no storage or nuclear-waste disposal problems. This satisfies Item (vi).

\section{Potential Future Applications}

While it appears quite feasible to build radiography facilities for special applications based on 14-MeV neutron generators, the real potential for this concept lies far in the future when intense sources of $14-\mathrm{MeV}$ neutrons will likely be available from plasma fusion machines associated with 
energy production. The distributed neutron-source intensities found in these fusion machines will be several orders of magnitude higher than even the most intense point-source $14-\mathrm{MeV}$ neutron generators currently available. In this scenario it would be appealing to divert some of the cooling water from these devices for use in radiography applications. Using specially designed water channels, shielding and detector arrays, it should be possible to perform quite sophisticated photon radiography measurements on bulk objects in reasonably short periods of time.

\section{Summary}

The present experiment demonstrates that photon radiography can be performed using highenergy gamma rays from the decay of 7.13 -second ${ }^{16} \mathrm{~N}$. This was accomplished by irradiating a loop of continuously flowing water with neutrons from a $14-\mathrm{MeV}$ neutron generator and then using this activated water in a simple radiography apparatus assembled at a remote location far from the neutron field. One-dimensional transmitted-photon scans were performed on four typical objects. One object was featureless while the remaining three possessed interesting features which could be identified quite readily in the generated radiographs. This method is flexible because of the inherent ability to adjust the intensity of the gamma-ray source by simply altering the coupling of the water line to the neutron field or adjusting the water flow rate. The system is inherently safe because after a few minutes the irradiated water is essentially benign and presents no storage or waste-disposal problems. While this method could be practical in special applications involving a $14-\mathrm{MeV}$ neutron generator, its real potential lies in the future when highly radioactive water obtained as a byproduct from cooling a D-T fusion reactor could be used to construct versatile photon radiography systems for applications 
which require highly penetrating, nearly monoenergetic photon sources.

\section{Acknowledgements}

This work was supported in part by the U.S. Department of Energy, Energy Research Programs, under contract W-31-109-Eng-38. One of the authors (DLS) is indebted to the Fusion Neutronics Laboratory, Department of Reactor Engineering, Tokai Establishment, Japan Atomic Energy Research Institute, for hosting his visit during January and February, 1994, when the present work was carried out. The skillful assistance of the Fusion Neutronics Laboratory technical staff during the course of this work is gratefully acknowledged.

\section{References}

[1] W.K. Roentgen, Sitzber. Wuerzberger Phys. - Med. Ges., December, 1895. Condensed English translations are given by W.F. Magie, Source Book in Physics, McGraw-Hill Book Company, New York (1935).

[2] C. Weyl and S.R. Warren, Jr., Radiologic Physics, 2nd Edition, Charles C. Thomas Publisher, Springfield, Illinois (1951) 
[3] A. Guinier and D.L. Dexter, X-Ray Studies of Materials, Interscience Publishers, New York (1963).

[4] B.E. Stern and D. Lewis, X-Rays, Sir Isaac Pitman and Sons, Ltd., Publishers, Bath, England (1970).

[5] H. Berger, "Radiographic Nondestructive Testing", Standardization News, Vol. 3, No. 3, p. 21, American Society for Testing and Materials, Philadelphia (1975).

[6] J.K. Tuli, Nuclear Wallet Cards, National Nuclear Data Center, Brookhaven National Laboratory, Upton, New York (1990).

[7] V. McLane, C.L. Dunford and P.F. Rose, Neutron Cross Sections, Vol. 2: Neutron Cross Section Curves, Academic Press, Inc., Boston (1988).

[8] E. Browne and R.B. Firestone, Table of Radioactive Isotopes, John Wiley \& Sons, New York (1986).

[9] S. Sato, Y. Seki, S. Mori, K. Maki and H. Takatsu, "Evaluation of Skyshine Dose Rate Due to Gamma-rays from Activated Cooling Water in Fusion Experimental Reactors", p. 946 Proceedings of the 8th International Conference on Radiation Shielding, American Nuclear Society, LaGrange Park, Illinois (1994). 
[10] R.D. Evans, The Atomic Nucleus, McGraw-Hill Book Company, Inc., New York (1955).

[11] E. Storm and H.I. Israel, Nuclear Data Tables, Vol. A7, 565 (1970).

[12] T. Nakamura et al., Proceedings of the 4th Symposium on Accelerator Science and Technology, 24-26 November 1982, Riken, Saitama, Japan, p. 155 (1982). 
Table 1: Transmission factors for $6-\mathrm{MeV}$ photons in slabs of pure-element materials ${ }^{2}$

\begin{tabular}{|c|c|c|c|c|c|c|}
\hline \multirow[b]{2}{*}{ Element } & \multirow[b]{2}{*}{$x(\mathrm{~cm})=$} & \multirow[b]{2}{*}{0.1} & \multicolumn{4}{|c|}{ Transmission $\left(I / / I_{0}\right)$} \\
\hline & & & 0.5 & 1.0 & 5.0 & 10.0 \\
\hline Carbon (C) & $=$ & 0.9944 & 0.9725 & 0.9457 & 0.7563 & 0.5720 \\
\hline Aluminum (Al) & & 0.9929 & 0.9649 & 0.9309 & 0.6992 & 0.4889 \\
\hline Iron $(\mathrm{Fe})$ & & 0.9763 & 0.8870 & 0.7868 & 0.3015 & 0.0909 \\
\hline Copper $(\mathrm{Cu})$ & & 0.9727 & 0.8706 & 0.7580 & 0.2502 & 0.0626 \\
\hline Lead $(\mathrm{Pb})$ & & 0.9518 & 0.7811 & 0.6102 & 0.0846 & 0.0072 \\
\hline
\end{tabular}

'Based on photon total cross sections from Storm and Israel [11] and pertinent material densities. 


\section{Figure Captions}

Figure 1: Schematic diagram showing the $14-\mathrm{MeV}$ neutron source, circulating water loop, water pump, water flow meter, and radiography apparatus employed in the present experiment (see Section 3.2).

Figure 2: Schematic diagram showing the radiography apparatus in greater detail, including the collimator assemblies, shielded water loop, sodium iodide scintillation detector and object to be studied (see Section 3.2).

Figure 3: Typical spectrum of gamma rays from ${ }^{16} \mathrm{~N}$ recorded with a sodium iodide scintillation detector and associated electronics instrumentation for pulse-height analysis (see Section 3.3).

Figure 4: Schematic diagram of Object " $A$ " investigated by radiography in the present experiment (see Section 4.1).

Figure 5: Schematic diagram of Object "B" investigated by radiography in the present experiment (see Section 4.1).

Figure 6: Schematic diagram of Object "C" investigated by radiography in the present experiment (see Section 4.1). 
Figure 7: Schematic diagram of Object " $D$ " investigated by radiography in the present experiment (see Section 4.1).

Figure 8: Results of one-dimensional photon scans of Objects "A", "B", "C", and "D" (see Section 4.3). 
Figure 1

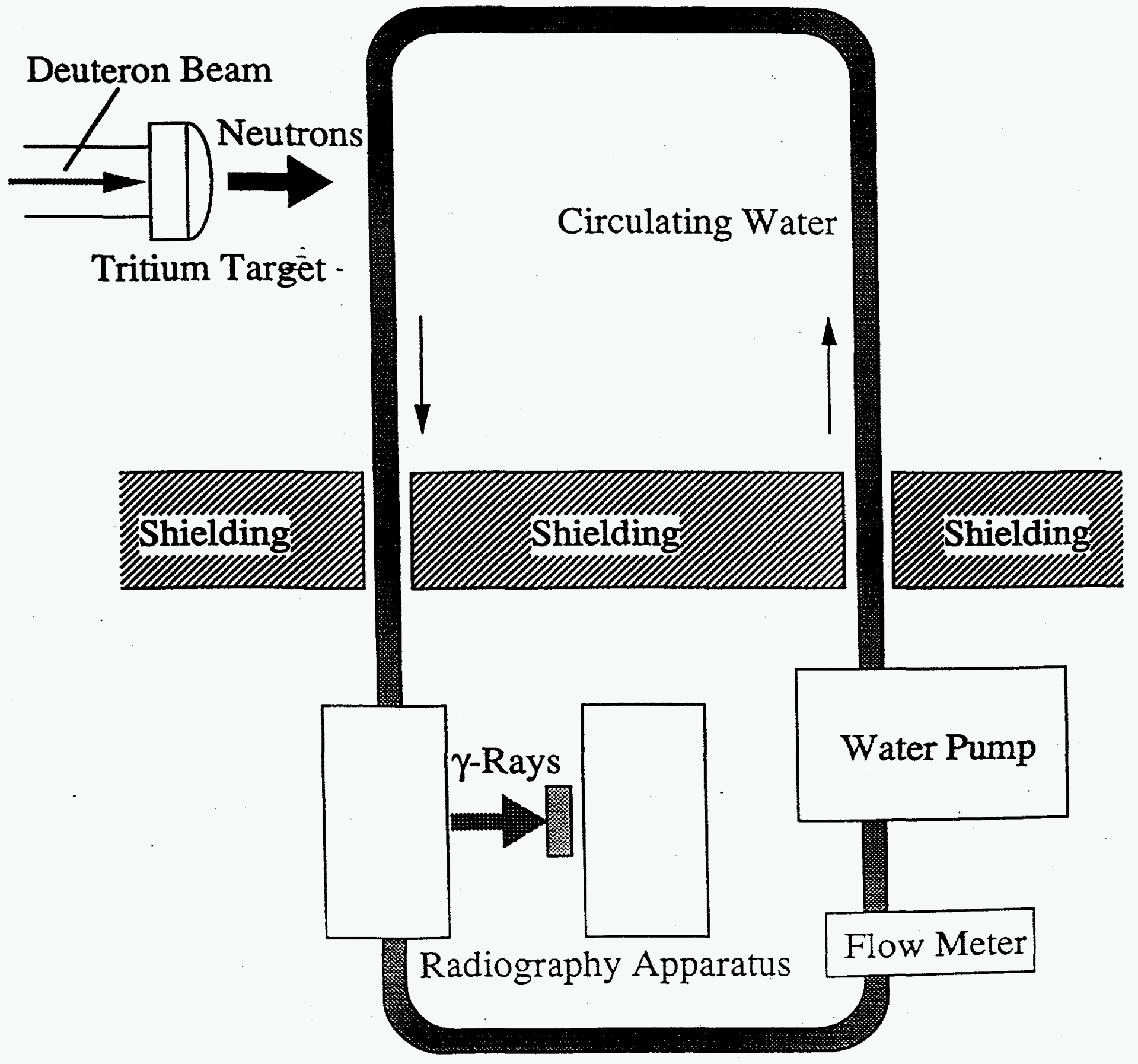




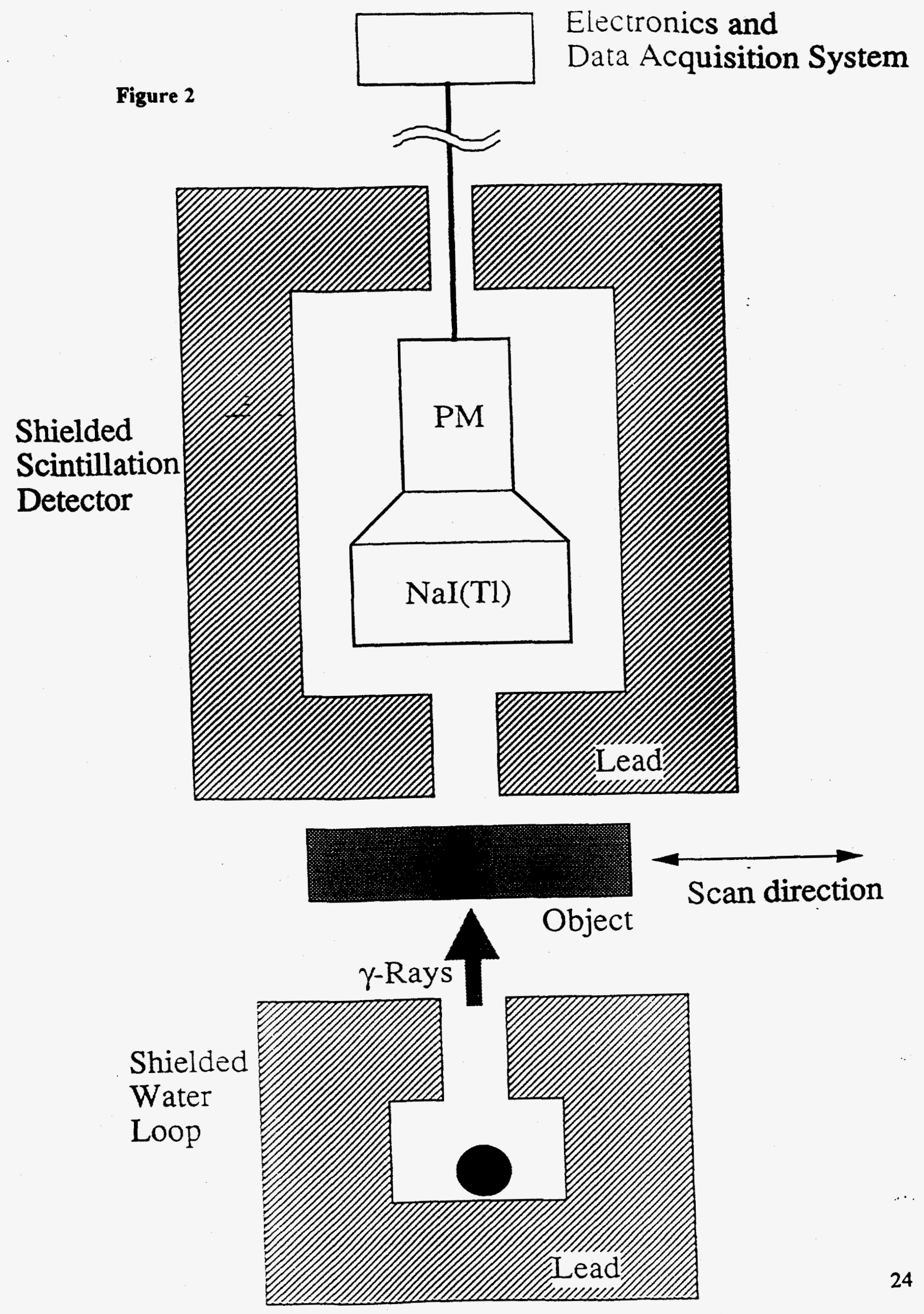


Figure 3

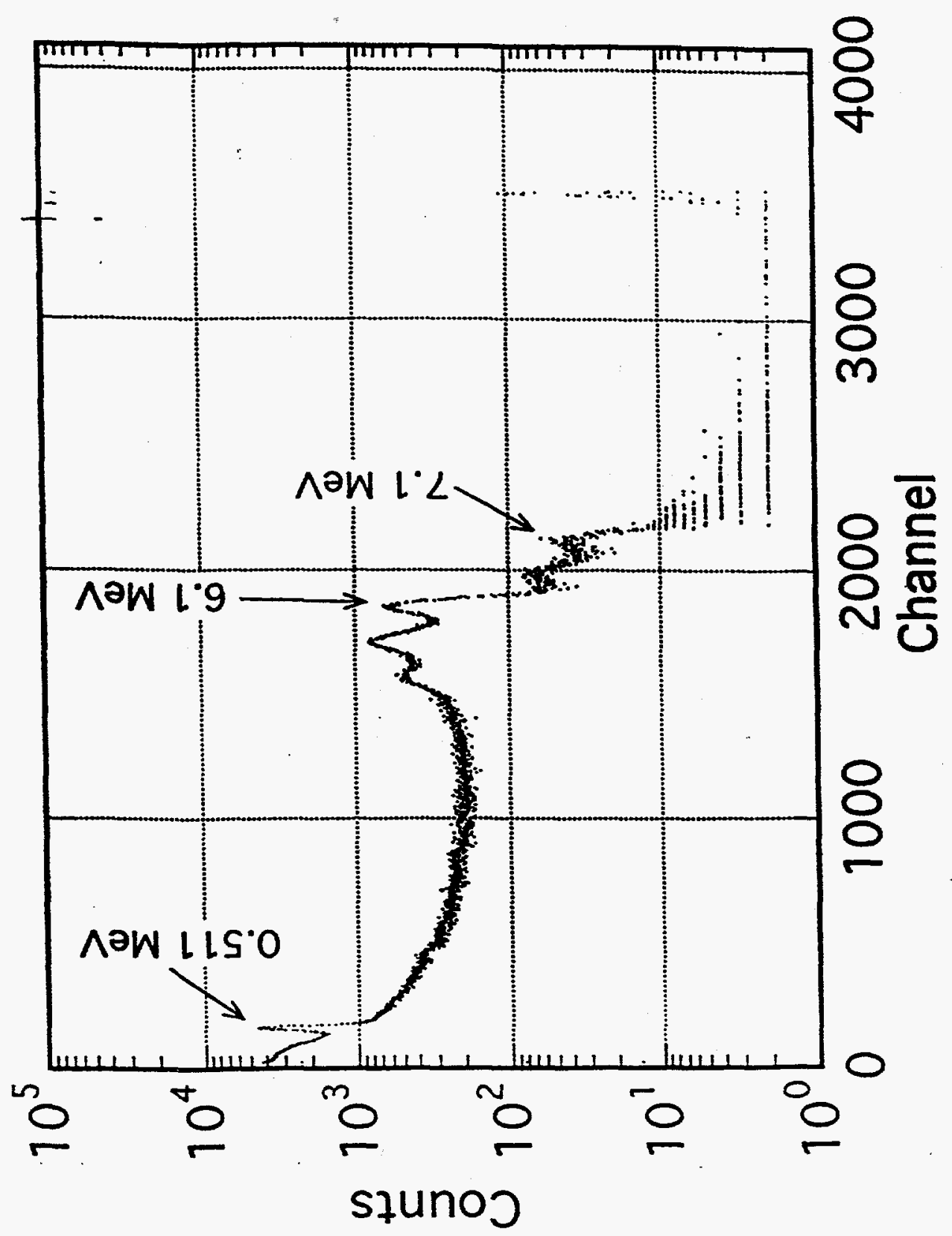


Figure 4

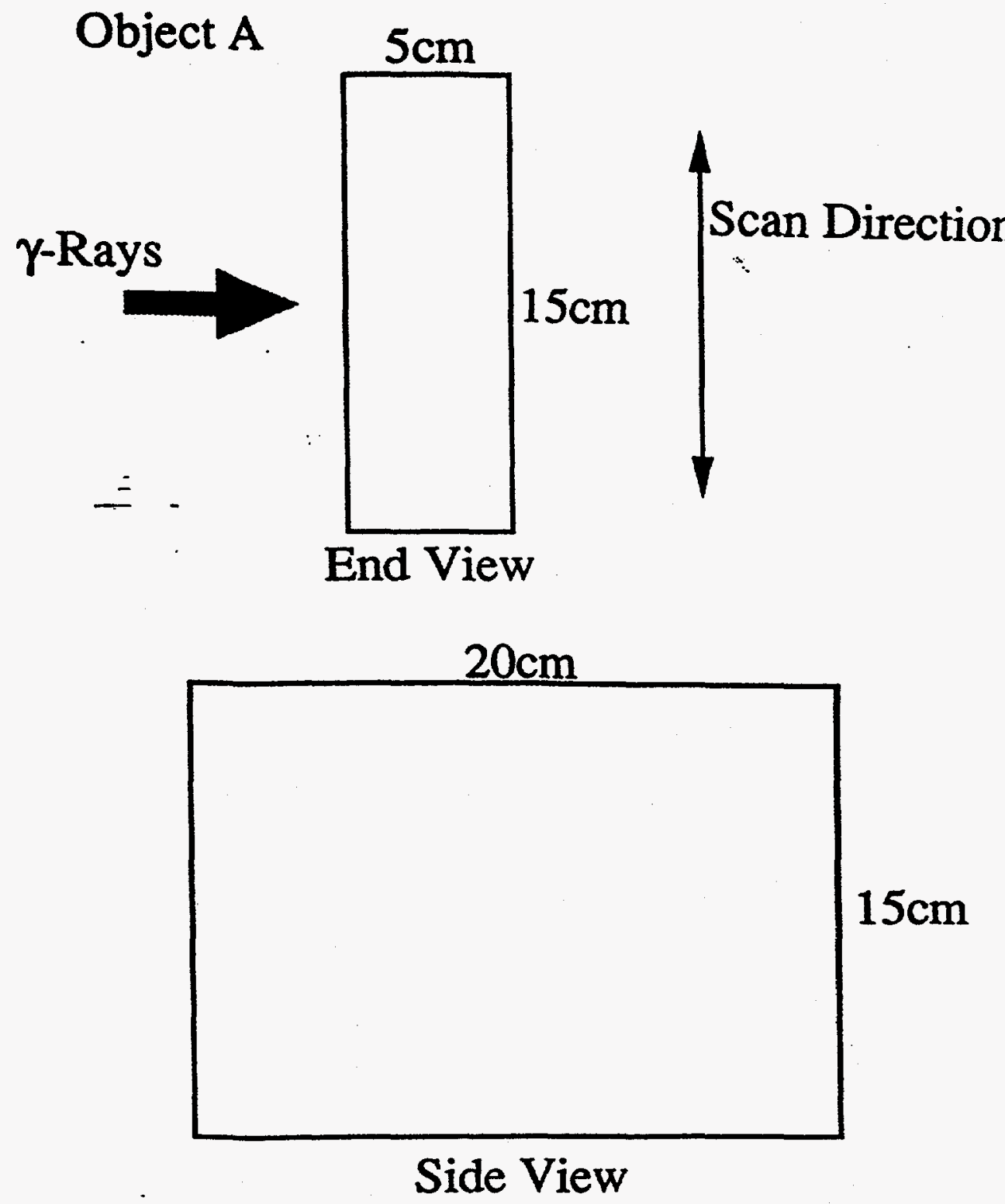


Figure 5

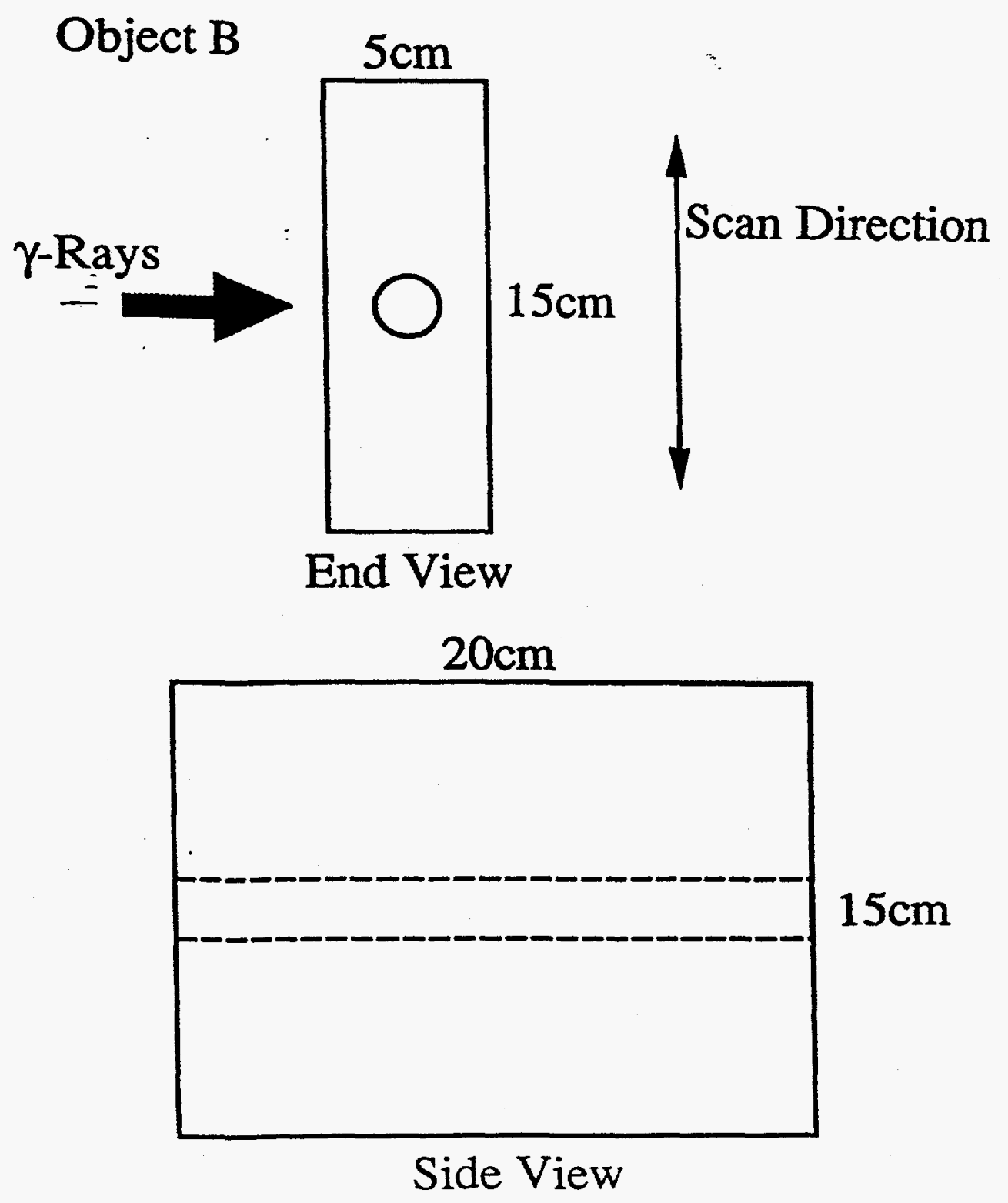


Figure 6
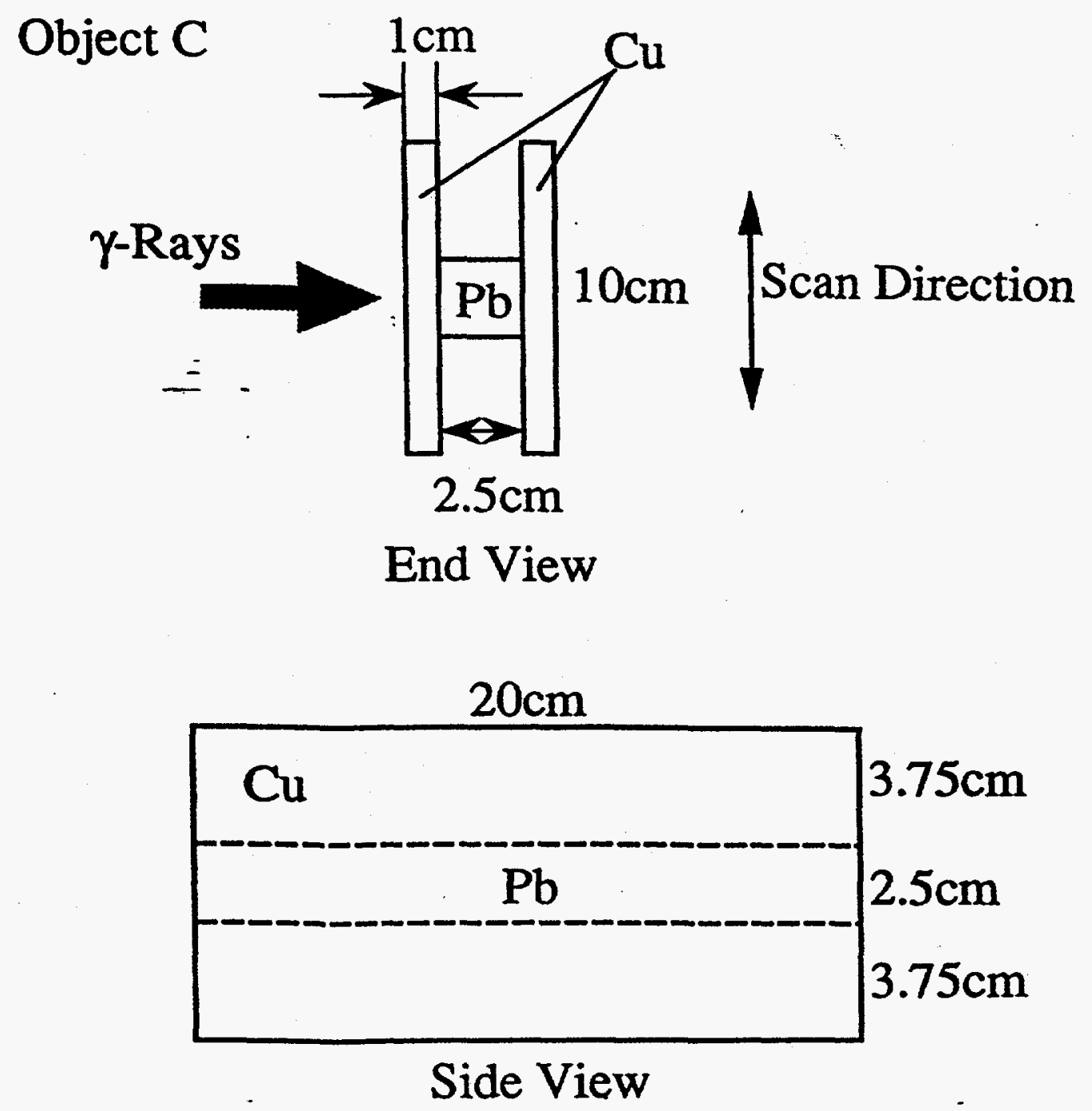
Object D

Figure 7
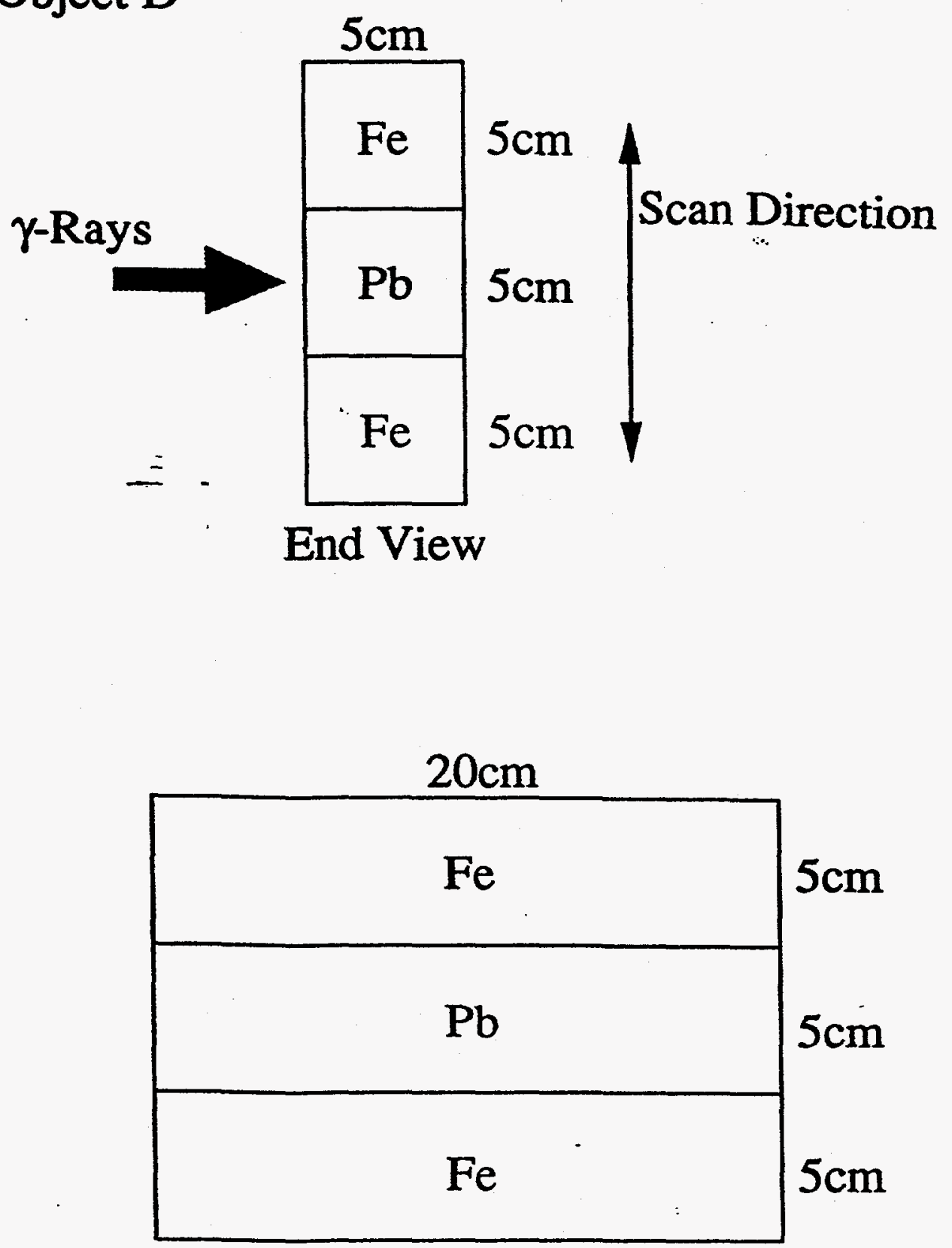

Side View 


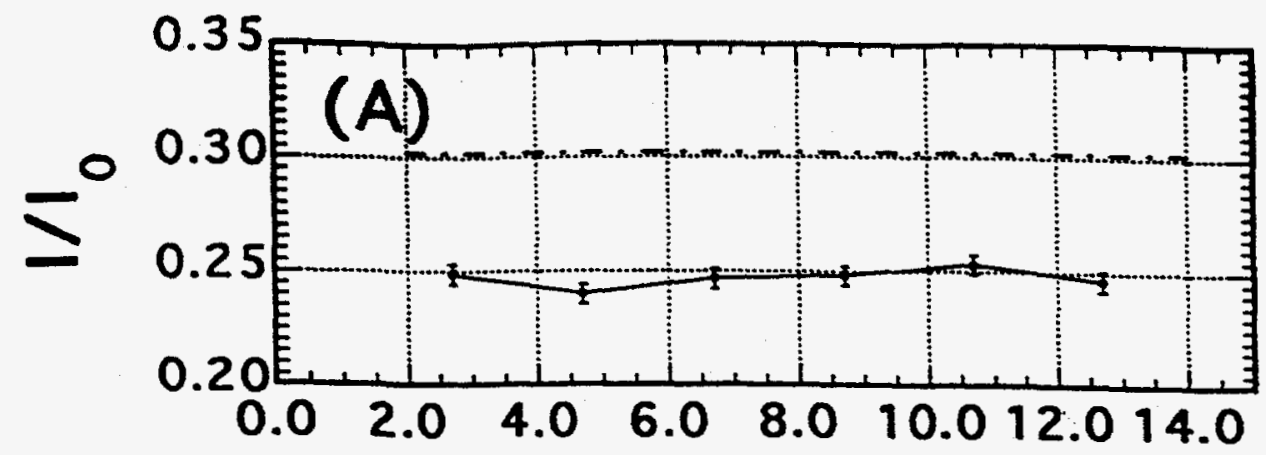

Figure 8
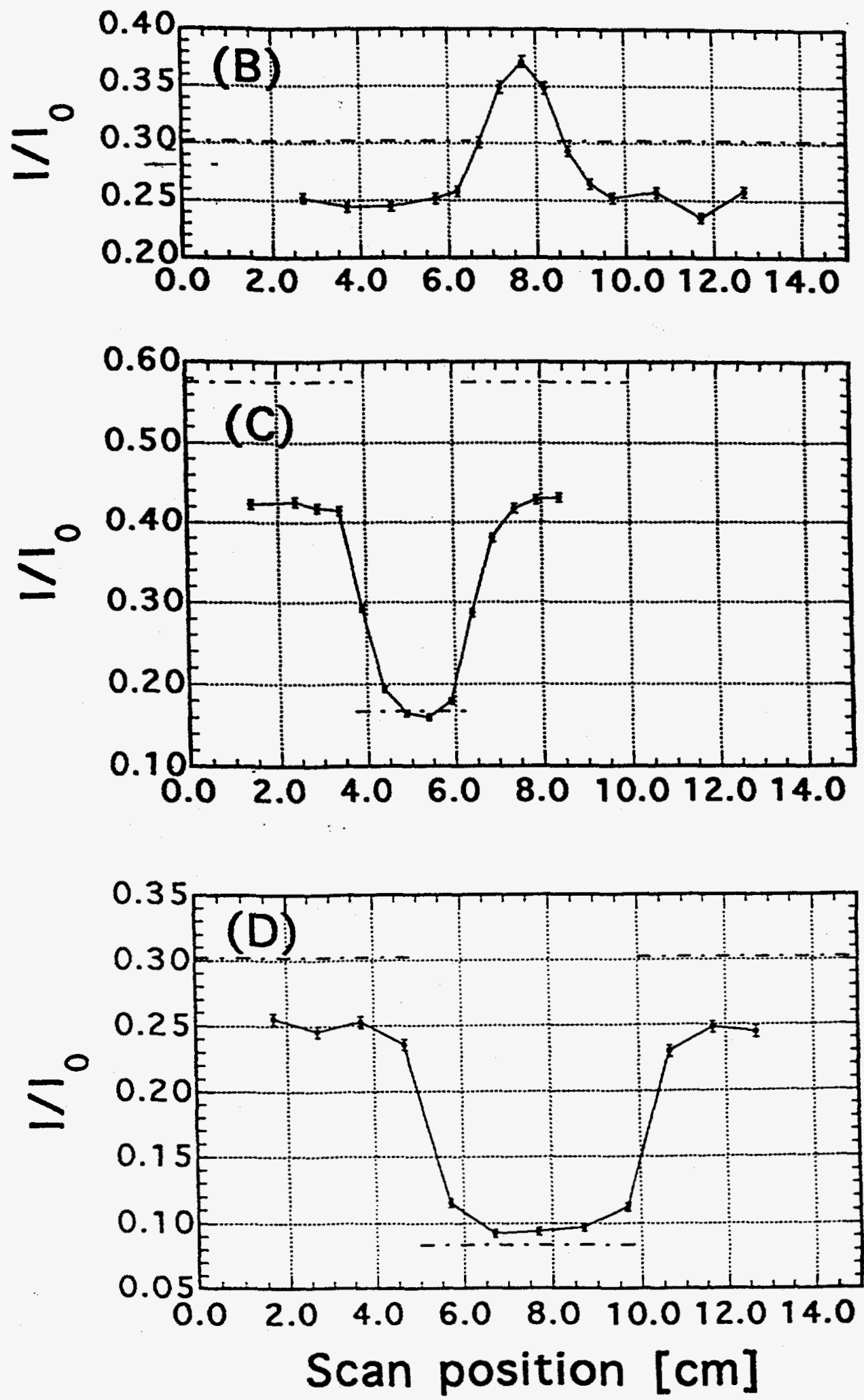